\title{
The Method and Application Prospect of the Non-Destructive Scientific and Technological Identification of Fake Antique Jade
}

\author{
Guyang Yang1, Huanhuan Wang² \\ ${ }^{1}$ School of Fine Art and Design, Shijiazhuang University, Shijiazhuang, China \\ ${ }^{2}$ The Invoice Museum, Hebei University of Economics and Business, Shijiazhuang, China \\ Email: yangguyang83@foxmail.com
}

How to cite this paper: Yang, G. Y., \& Wang, H. H. (2022). The Method and Application Prospect of the Non-Destructive Scientific and Technological Identification of Fake Antique Jade. Archaeological Discovery, 10, 60-67.

https://doi.org/10.4236/ad.2022.101003

Received: December 24, 2021

Accepted: January 14, 2022

Published: January 17, 2022

Copyright $\odot 2022$ by author(s) and Scientific Research Publishing Inc. This work is licensed under the Creative Commons Attribution International License (CC BY 4.0).

http://creativecommons.org/licenses/by/4.0/

\begin{abstract}
Modern fake antique jade is a means of rapid aging to make its appearance similar to excavated ancient jade, which seriously disrupts the orderly development of the collection market. This occurs because the current identification method is still based on traditional ophthalmic identification, which relies too much on the subjective consciousness of the appraiser. At present, scientific and technological testing and identification has become an important part of cultural relics identification, and promoting the development of jade non-destructive scientific and technological identification can continuously improve the jade identification system. In view of this, we discussed the application methods and feasibility of nondestructive scientific and technological identification in the four aspects of traditional ophthalmic identification: material identification, qualitative change characteristic identification, exogenous substance identification, and process trace identification. We found that non-destructive technology identification has broad prospects for development in the field of jade identification.
\end{abstract}

\section{Keywords}

Fake Antique Jade, Non-Destructive, Scientific and Technological Identification, Improve the Identification System

\section{Introduction}

Since the Neolithic period, China has had a long history of using jade for nearly 10,000 years, and during this long process jade has been given many unique humanistic meanings, giving rise to a unique Chinese jade culture. The ancient jade collection market has flourished in recent years. Driven by interest, a large 
number of antique jade imitations have emerged, which have seriously disrupted the collecting market, causing collectors to suffer economic losses as well as undermining the orderly transmission of traditional jade culture. In recent years in particular there have been many controversial and overpriced jade collections in the auction market, such as the once widely speculated "jade clothes sewn with gold wire" and "jade stool from the Han dynasty", which have also highlighted the confusion in the field of collecting and identification. The "jade clothes sewn with gold wire" was valued at 2.4 billion yuan and mortgaged 700 million yuan from the banks by the collecter, which is a modern imitation. Similarly, the "jade stool from the Han dynasty" was auctioned for 220 million yuan, is also a modern imitation. These have also attracted the attention of the relevant authorities, with CCTV reporting on the chaos of faking and identification of antique jade. The main reason for this chaos is that jade identification is still mainly ocular, which is limited by differences in the knowledge and experience of the appraiser, resulting in results that are often only the opinion of the appraiser, and there is an urgent need for modern technological testing methods to intervene in the field of appraisal, combining technological appraisal with ocular appraisal to make appraisal more scientific and standardised (Wang, 2015; Wang, 2020; Huang, 2019).

Technology identification is the identification of the age, origin and authenticity of cultural objects through modern scientific theories, techniques and methods, and has developed rapidly in recent years, mainly in the fields of ceramics and bronzes, where the means of scientific and technological analysis are relatively mature. However, there is still a lack of systematic research into the scientific and technical identification of antique jade imitations, and there are no well-developed scientific and technical identification procedures. In addition, a large number of modern methods of scientific and technological analysis are destructive (Gan, 2008), all of which can cause irreversible damage to the antique, especially in the collecting world, where no holder wants the objects in their collection to suffer damage, which requires the use of destructive analysis to be avoided as far as possible in the scientific and technological identification of jade objects. If some rapid, accurate and non-destructive methods can be found for the identification of antique jade imitations, it will not only reduce the losses of collectors, but will also contribute greatly to the standardization of the collecting market. With the improvement of instrument accuracy, some non-destructive technologies have begun to be more and more applied to the field of cultural relics testing. Zhao and Gan (2009) used Raman spectroscopy to analyze the characteristics of jade from different origins. Zhao et al. (2020) used EDXRF to analyze that black mercury Qin was caused by cinnabar. Using Raman spectroscopy, Chen et al. (2007) found that jade heated at high temperatures had different spectral characteristics. Their works have a very important enlightening effect on the non-destructive identification of jade.

According to the traditional jade identification with ocular from the four as- 
pects of material identification, identification of quality changes, identification of exogenous substances and identification of craft marks, this paper will discuss the non-destructive identification method and its application prospect of fake antique jade.

\section{Jade Material Identification}

Jade objects are carved from raw jade through a process in which only the appearance of the raw material is changed. The mineral composition of the processed jade is the same as that of the raw jade, and material identification means that the jade is analyzed and tested by instruments to determine its mineral composition. Material analysis is the basis for the identification of modern antique jade imitations, and only by understanding the material of the jade can we provide a basis for subsequent testing and analysis. Although a large number of our ancient jades were made of tremolite nephrite, the material used in different eras or regions still varies, so material analysis can be used to identify whether or not it matches the chronological and regional characteristics displayed by its shape. At the same time, the price of tremolite jade, as represented by Hetian jade, is still high, and common forgers use ordinary stones with a similar appearance to tremolite jade for profit, and then "grey skin" and "colouring", which even an experienced person cannot detect with the naked eye, whereas modern technological testing methods can immediately identify the material.

With the development of non-destructive analytical methods such as diffuse reflectance infrared spectroscopy (DRIFT) and Raman spectroscopy (Raman) in recent years, these two analytical methods have gradually become common in the identification of excavated ancient jade materials (Zhao \& Gan, 2009; Li et al, 2013). Both diffuse reflectance infrared spectroscopy and Raman spectroscopy are fast and non-destructive, while with the development of technology, both testing instruments are now available as hand-held portable devices, which are not limited by the appearance of the size and shape of the sample, and have a broad future in the material identification and analysis of antique jade imitations.

\section{Identification of Quality Changes in Jade}

In the antique collecting world, the so-called quality changes in jade do not refer to changes in the mineral structure of the material, but rather to changes in the appearance of the material, such as "gray skin" and "coloring", that occur during the burial process when the jade is affected by its surroundings. These changes are the result of the long period of time that the jade was buried and affected by the surrounding environment, modern antique jade imitations has been made to look similar to unearthed jade by burning, soaking in acid and alkaline solutions, and other means. The following is an example of the use of nondestructive technology in the identification of "chicken bone white" (Figure 1) and "mercury percolation" (Figure 2). 


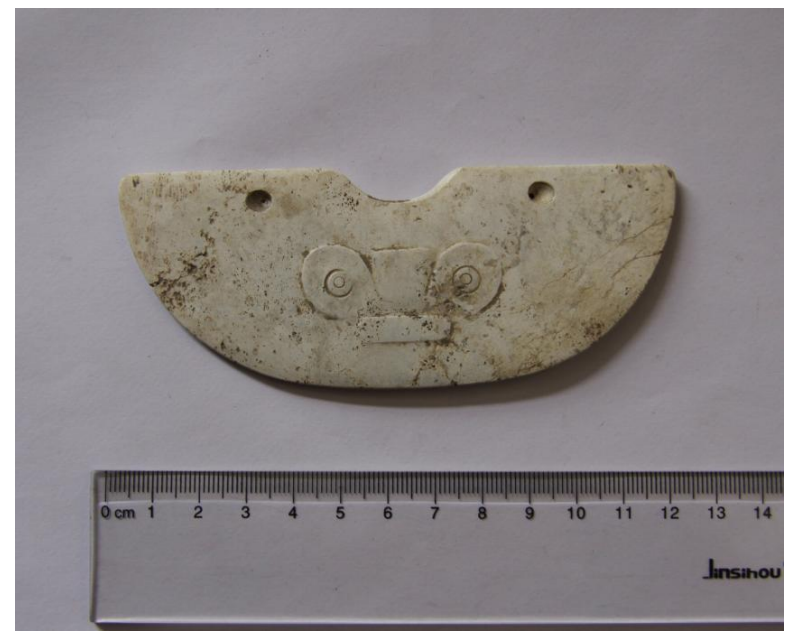

Figure 1. Chicken bone white jade.



Figure 2. Mercury percolation jade.

"Chicken bone white" is a common type of modern antique fake quality change, although there is still no consensus in the academic community on the formation of excavated "chicken bone white" jade, however, the mineral characteristics have not changed from the current study of the material (Zhu et al., 2002), while modern imitations of "chicken bone white" are usually whitened by high-temperature heating. Figure 3 shows the appearance changes of original jade and after heating at $1000^{\circ} \mathrm{C}$. The heated jade will change in the spectral peaks by Raman spectroscopy, and the spectral peaks of some characteristic points will weaken or even disappear, while there is no such spectral change in the excavated jade. This phenomenon can effectively identify whether the jade has been heated at high temperature or not (Chen et al., 2007).

The "mercury percolation" is a black percolation formed when the jade is infiltrated by surrounding materials during the burial process, and studies have 


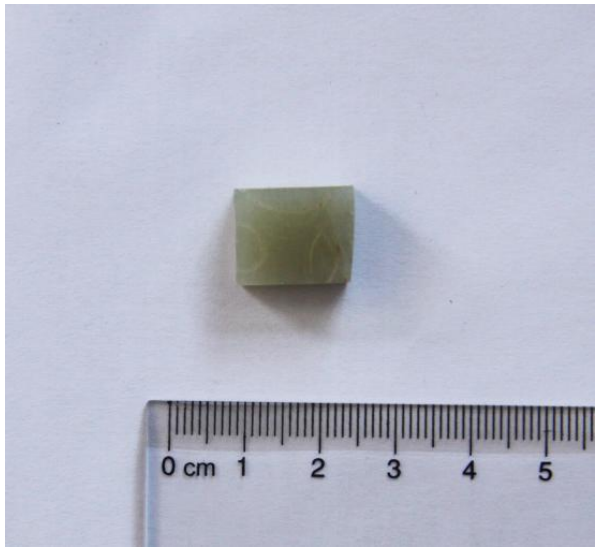

(a)

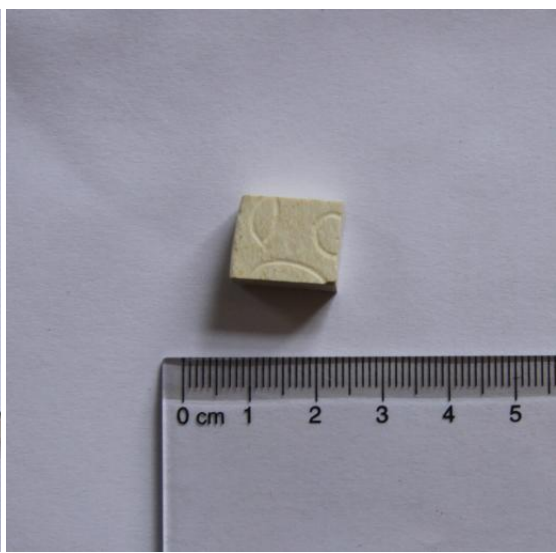

(b)

Figure 3. Appearance changes of original jade and after heating at $1000^{\circ} \mathrm{C}$. (a) Original jade; (b) After heating at $1000^{\circ} \mathrm{C}$.

shown that this black percolation is caused by the surrounding mercury sulfide infiltration of the jade during the burial process (Zhao et al., 2020). Modern imitations of "mercury percolation" usually use stone stains for dyeing, but these stains are organic chemical synthetics, which do not contain the element Hydrargyrum. Therefore, the detection of the elemental composition of the percolation can effectively identify the "mercury percolation", and the use of energy dispersive X-ray fluorescence spectroscopy (EDXRF) can detect mercury in the black percolation of excavated ancient jade, while the black percolation of modern imitations is dyed with modern chemicals, which is not detected by EDXRF. This indicates that the black "mercury percolation" can be non-destructively identified by EDXRF. EDXRF has been widely used in the field of elemental composition testing of inorganic materials such as ceramics and bronzes, which is fast and non-destructive.

From the above study of "chicken bone white" and "mercury percolation", we can see that the nondestructive identification of jade texture change characteristics means that the elements and mineral structure of the textural parts are examined by instruments, and the results are analyzed to determine whether the textural changes are made by modern imitation. Usually forgers imitate ancient jade from before the Ming dynasty with "gray skin" and "color", this is due to the fact that there are few heirlooms of ancient jade from before the Ming dynasty, and the production of texture change characteristics makes it have the feeling of so-called excavation, therefore, the scientific and technical inspection of the quality change area plays a very important role in the nondestructive testing of ancient jade.

\section{Identification of Exogenous Substances of Jade}

Exogenous Substances is the material attached to the surface of jade mineral material. It can be divided into visible exogenous substances and invisible exogenous substances. Visible exogenous substances are the material contaminated 
by other surrounding buried materials in the process of jade burial like cinnabar (Figure 4).

Ancient jade excavated from the earth has undergone a process of weathering, leaching, and osmotic cementation due to the burial process in the surrounding environment (Feng et al., 2005). This is a common feature of buried ancient jade, which often forms a glass-like "crust" on the surface of the object. Modern forgers often paint the surface of jade with waxes, lacquers, epoxy resins and other organic substances to imitate this "crust". Waxes, lacquers, epoxies, etc. are all exogenous substances that are not minerals of the jade itself, with waxes being the most common due to their ease of use and technical maturity. The surface of waxed jade has a glassy "crust" that is very similar to that of excavated jade and is very difficult to identify with the naked eye. At present, scholars have studied the waxing process of antique jade by infrared spectroscopy, and found the unique spectral peaks of wax substances, but it uses the potassium bromide powder press method, which requires direct sampling on the sample, although the amount of sample required is small but still destructive analytical, and the experimental process is fine and complex, which is suitable for scientific research, but the practicality is poor in identification and testing, not conducive to large-scale promotion in the actual identification. With the development of modern technology, current infrared spectroscopy inspection instruments can be used to achieve direct detection by adding diffuse reflection accessories, no longer need to carry out sample preparation, so that the purpose of nondestructive testing is also achieved. At the same time, handheld portable diffuse reflectance infrared spectrometers are now more and more accurate, so that samples that are too large to fit into the sample compartment can also be detected quickly and non-destructively. As the instrument becomes more popular, the detection of exogenous substances will become more convenient and accurate.

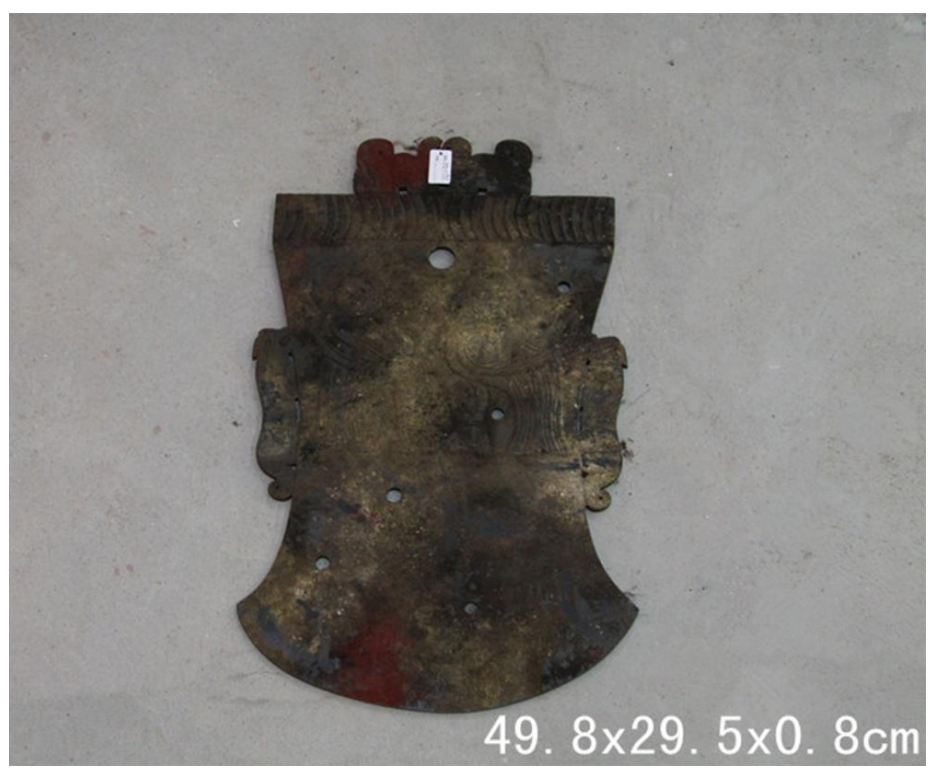

Figure 4. Jade with cinnabar. 


\section{Identification of Craft Marks of Jade}

Because of the differences between ancient jade carving techniques and modern machine carving processes, the processing marks left on the jade are also different. The analysis of jade craft marks is to observe and analyze the surface of the object such as cutting, polishing, drilling, shading lines and other processing marks to determine whether the processing characteristics are of the age that matches its ornamental characteristics. At present, scholars have made some progress in this area by means of micro-CT and super depth-of-field microscopy, but this method also has certain limitations. Firstly, this method needs to compare a large number of ancient processing marks with modern processing marks, which requires a lot of preliminary work. Secondly, the analysis of craft marks cannot distinguish modern jade imitations that made by the ancient method, although the ancient method is time-consuming and labor-intensive, it cannot be ruled out that some high-end fine imitators use the ancient manual method to produce in order to obtain higher benefits. Thirdly, not all jade can be easily observed for processing marks, such as plain jade. Although there are certain limitations in the analysis of craft marks, this method is still practical for most jade, and more in-depth research is needed to identify modern processing marks.

\section{Conclusion}

As can be seen from the above, the scientific and technical identification of antique jade can be carried out through four aspects of material identification, identification of quality changes, identification of exogenous substances and identification of craft marks, thus allowing a more comprehensive analysis of the identified jade. With the continuous development of non-destructive scientific testing technology, the scope of application of excavated ancient jade testing has gradually expanded, and there will be more data of unearthed ancient jade for comparison when identifying fake antique jade. At the same time, with the progress of science and technology, more and more testing methods are available for non-destructive analysis test, the accuracy of equipment continues to improve, more instruments are hand-held and portable etc., so the scientific and technological identification of fake antique jade will have broad prospects for development.

\section{Acknowledgements}

This work is financially supported by the Shijiazhuang University Scientific research start-up project No. 17QN001 "Nondestructive identification method research on fake antique jade with surface waxing".

\section{Conflicts of Interest}

The authors declare no conflicts of interest regarding the publication of this paper. 


\section{References}

Chen, X. R., Guo, S., Zhang, W., \& Xue, L. (2007). Application of Raman Spectroscopy in Antique Jade Identification. Sciences of Conservation and Archaeology, No. 1, 43-45+ 74.

Feng, M., Zhang, J. G., Wang, R., Wang, C. S., \& Gong, M. (2005). Analysis of Lingjiatan Ancient Jade Qin Process. Sciences of Conservation and Archaeology, No. 1, 22-26.

Gan, F. X. (2008). Some Views on the Scientific and Technological Archaeological Research of Ancient Chinese Jade and Jade. Sciences of Conservation and Archaeology, $20,17-26$.

Huang, Y. F. (2019). The Necessity and Specific Application of Modern Science and Technology. Sciences of Conservation and Archaeology, No. 11, 103.

Li, J. J., Yang, X. L., Liu, X. W., \& Li, D. M. (2013). Application of Infrared Detection Technology in the Identification of Ancient Jade. Infrared, 34, 34-38.

Wang, C. S. (2015). Current Situation, Problems and Prospects of Cultural Relics Science and Technology Identification. Cultural Relics World, No. 12, 52-55.

Wang, S. Q. (2020). Exploration on the Application of Modern Technology in Cultural Relics Identification. Identification and Appreciation of Cultural Relics, No. 5, 91-93.

Zhao, H. X., \& Gan, F. X. (2009). Raman Spectroscopic Analysis of Soft Jade from Different Origin and Its Application in Nondestructive Research of Ancient Jade. The Journal of Light Scattering, 21, 345-354.

Zhao, R. T., Yu, P., Shao, F., Li, Y. L., \& Zhang, X. L. (2020). The Phenomenon of Black Mercury Silver Qin Unearthed from Noble Tombs in Hunan Warring States Period. Museum, No. 2, 97-101.

Zhu, Q. W., Zhang, J. G., \& Wu, M. (2002). Study on the Jade Quality of White Jade Unearthed from Lingjiatan. Journal of Rock Mineralogy, S1, 129-133. 